\title{
Tarsal tunnel syndrome in patients with fibromyalgia
}

\author{
Yoon-Sik Jo' ${ }^{1}$, Bora Yoon ${ }^{2}\left(\mathbb{D}\right.$, Jun Yeong Hong ${ }^{2}\left(\mathbb{D}\right.$, Chung-Il Joung ${ }^{(\mathbb{D}}$, \\ Yuseok Kim² ${ }^{2}$, Sang-Jun $\mathrm{Na}^{2} \mathbb{D}$ \\ ${ }^{1}$ Department of Neurology, Konkuk University School of Medicine, Chungju, South Korea \\ ${ }^{2}$ Department of Neurology, Konyang University College of Medicine, Daejeon, South Korea \\ ${ }^{3}$ Department of Internal Medicine, Konyang University College of Medicine, Daejeon, South Korea
}

\begin{abstract}
Objectives: This study aims to evaluate the frequency of tarsal tunnel syndrome (TTS) in fibromyalgia (FM) patients.

Patients and methods: In this prospective study, we investigated paresthesia of the foot, sensory and motor deficits, atrophy of the abductor hallucis muscle, and the presence of Tinel's sign in 76 female FM patients (mean age $39.3 \pm 7.4$ years; range, 24 to 52 years) and 60 sex-matched healthy control subjects (mean age 38.6 \pm 8.2 years; range, 28 to 49 years) without FM between July 2016 and June 2018. Bilateral electrophysiological studies of the tibial, peroneal, sural, and medial as well as lateral plantar nerves were performed.

Results: Paresthesia was observed in 22 FM patient extremities and four control subject extremities $(p=0.002)$. Local tenderness at the tarsal tunnel was observed in $12 \mathrm{FM}$ patient extremities and two control subject extremities $(p=0.021)$. TTS was detected electrophysiologically in $14 \mathrm{FM}$ patient extremities and two control subject extremities $(p=0.009)$.

Conclusion: Paresthesia of the foot and local tenderness at the tarsal tunnel were significantly more prevalent in FM patients than in healthy control subjects. TTS is statistically more frequent in patients with FM than the normal population. The potential comorbidities of TTS and paresthesia of the foot should be carefully examined in FM patients.

Keywords: Carpal tunnel syndrome, electrodiagnosis, fibromyalgia, nerve compression syndrome, paresthesia, tarsal tunnel syndrome.
\end{abstract}

Fibromyalgia (FM) is a chronic disorder characterized by widespread musculoskeletal pain and tender points, complaints of paresthesia, sleep disturbances, morning stiffness, fatigue, irritable bowel syndrome, and frequent psychological distress. ${ }^{1}$ Paresthesia of the foot is particularly problematic in FM patients; moreover, Tinel's sign is often positive in those patients. ${ }^{2}$ The cause of paresthesia in FM patients remains poorly understood; however, paresthesia is thought to result from abnormal sensory perceptions elicited by central sensitization in FM. ${ }^{3,4}$ To date, the role of peripheral nervous system (PNS) in paresthesia as a component of FM has not yet been defined.
Paresthesia has been reported in 26-84\% FM patients making the diagnosis of associated entrapment neuropathies difficult in FM patients. ${ }^{5}$ In clinical practice, it is important to distinguish between the symptoms of FM and entrapment neuropathies, which are easily overlooked or misdiagnosed in FM patients. Therefore, the association between carpal tunnel syndrome (CTS), one of the relatively common entrapment neuropathies, and FM has been studied extensively. However, in practice, paresthesia of the foot is also not uncommon. Therefore, we conducted a study to compare the symptoms of tarsal tunnel syndrome (TTS), one of the

Received: December 06, 2019 Accepted: July 06, 2020 Published online: January 14, 2021

Correspondence: Sang-Jun Na, MD. Department of Neurology, Konyang University College of Medicine, 35365 Daejeon, South Korea. Tel: +82-43-840-8232 e-mail: nukedoc@hanmail.net

\section{Citation:}

Jo YS, Yoon B, Hong JY, Joung Cl, Kim Ş, Na SJ. Tarsal tunnel syndrome in patients with fibromyalgia. Arch Rheumatol 2021;36(1):107-113.

This is an open access article under the terms of the Creative Commons Attribution-NonCommercial License, which permits use, distribution and reproduction in any medium, provided the original work is properly cited and is not used for commercial purposes (http://creativecommons.org/licenses/by-nc/4.0/) 
entrapment neuropathies in which patients may complain of paresthesia of the foot, in patients with and without FM. In addition, we studied the relationship between TTS and FM, which has very rarely been investigated. Here, the American College of Rheumatology (ACR) 1990 FM classification criteria were used to investigate objectively the measured objective clinical findings rather than subjective symptoms. Thus, in this study, we aimed to evaluate the frequency of TTS in FM patients.

\section{PATIENTS AND METHODS}

Seventy-six consecutive female patients (mean age $39.3 \pm 7.4$ years; range, 24 to 52 years) who received a diagnosis of FM according to the ACR 1990 criteria for the classification of FM between July 2016 and June 2018 were enrolled in this prospective study conducted at Konyang University Hospital. All patients were homemakers. Sixty age- and sex-matched healthy volunteers (mean age 38.6 \pm 8.2 years; range, 28 to 49 years) were recruited from the hospital staff as control subjects. The study protocol was approved by the Konyang University Medical Faculty Ethics Committee (approval no: KYUH 2016-06-022). A written informed consent was obtained from each participant. The study was conducted in accordance with the principles of the Declaration of Helsinki.

The following exclusion criteria were applied: (i) patients $<18$ years or $>65$ years of age; (ii) history of degenerative bone disease; (iii) connective tissue disorder such as rheumatoid arthritis; (iv) certain systemic disorders including diabetes mellitus and hypothyroidism; $(v)$ certain foot deformities, ankle sprains, or fractures; (vi) varicose veins or other unusual mass lesions (such as a schwannoma or ganglion cyst in the tarsal tunnel); (vii) medication history that might have triggered neuropathy; (viii) history of receiving local steroid injection; and (ix) use of anti-epileptic drugs to control neuropathic pain. No FM patients or control subjects were engaged in an occupation or activity involving repetitive weight bearing, such as jogging. Patients and controls were paired according to age $(p=0.65)$, height $(p=0.74)$, weight $(p=0.69)$, and body mass index $(p=0.69)$.
Tests were performed on 152 extremities in $76 \mathrm{FM}$ patients and on 120 extremities in 60 control subjects. Paresthesia including numbness and tingling sensations of the foot, sensory deficits including hypoesthesia in the plantar nerve dermatome, local tenderness at the tarsal tunnel, weakness of flexion of the toe, and atrophy of the abductor hallucis muscle were recorded. Tinel's sign (used in the diagnosis of TTS) was evaluated. To test for Tinel's sign, we tapped the posterior tibial nerve of the tarsal tunnel and recorded the sign as positive if the patient perceived paresthesia that radiated distally. ${ }^{4}$

Electrophysiological studies were performed by a well-trained neurophysiology technician using a Sierra ${ }^{\circledR}$ II Wedge electroneuromyography equipment (Kennewick, Washington, USA). According to the American Association of Neuromuscular \& Electrodiagnostic Medicine guidelines, electrophysiological studies were performed. ${ }^{6}$ Patients with a clinical diagnosis of TTS as evidenced by paresthesia in the territory of the tibial nerve and compatible nerve conduction study (NCS) abnormalities were included.

Abnormalities in NCSs consisted of delayed distal motor latencies (DMLs) of the tibial nerve (95\% confidence interval [CI]: $\geq 4.6 \mathrm{~ms}$ ) or medial/lateral plantar nerve conduction amplitude outside of our laboratory reference ranges (95\% CI: $\leq 31 \mu \mathrm{V}$ for medial plantar amplitude, 95\% $\mathrm{CI}: \leq \mathrm{I} 1 \mu \mathrm{V}$ for lateral plantar amplitude). In the case of prolonged DMLs of the posterior tibial nerve, DMLs of the fibular, median, and ulnar nerves were carefully examined. However, in the case of generalized prolongation of DMLs, the subject was excluded to avoid inclusion of patients with chronic/acute inflammatory demyelinating neuropathy, multifocal motor neuropathy with multiple conduction blocks, hereditary sensorimotor neuropathy, and distal acquired demyelinating symmetric neuropathy.

The NCSs involved measurement of the amplitude of sensory nerve action potentials, sensory nerve conduction velocity, DML of the medial and lateral plantar nerves, and motor nerve action potentials. The motor nerve action potentials of both feet were also measured and compared. The difference between DML values of the medial and lateral plantar nerves was also 
determined. An amplitude of sensory nerve action potentials of $>5 \mathrm{mV}$, sensory nerve conduction velocity of $>40 \mathrm{~m} / \mathrm{sec}$, DML of the medial plantar nerve to abductor hallucis muscle of $<6.2 \mathrm{~m} / \mathrm{sec}$ and DML of the lateral plantar nerve to the abductor digiti quinti muscle of $<7 \mathrm{~m} / \mathrm{sec}$, and an amplitude of motor nerve action potentials of $>5 \mathrm{mV}$ were considered normal in our laboratory. A difference of $1 \mathrm{~m} / \mathrm{sec}$ between the DML values of for the medial and lateral plantar nerves was accepted to indicate TTS.

Electrophysiological diagnosis of TTS was based on slowed conduction velocities across the tarsal tunnel, and/or small amplitudes, or absence of response demonstrated by the medial or lateral sensory nerves. ${ }^{6,7}$

\section{Statistical analysis}

The sample size calculation was based on the preliminary study of the frequency of TTS in FM patients. ${ }^{8}$ We calculated the sample size using the difference in mean and standard deviation with a power of $80 \%$ in the preliminary study. The reasonable sample size was 55 patients.

All statistical analyses were performed using the IBM SPSS version 21.0 (IBM Corp., Armonk, NY, USA). To evaluate the differences in the data presented as mean values between the groups, Student's t-tests were used. The chi-squared test or Fisher's exact test was performed to compare the data expressed as percentage between the groups. A $p$ value $<0.05$ was considered statistically significant.

\section{RESULTS}

The demographic characteristics of the two groups are summarized in Table 1. Disease duration ranged from 6 to 42 months in FM patients. There were no significant differences between the two groups in terms of age, height, or weight ( $>0.05$ for all comparisons).

Clinical and electrophysiological characteristics of both groups are shown in Table 2. Self-reported paresthesia such as numbness and tingling sensations of the foot was identified for 22 (14.5\%) FM patient extremities versus four (3.3\%) control subject extremities $(p=0.002)$. Twelve (7.9\%) FM patient extremities and two (1.7\%) control subject extremities had local tenderness at the tarsal tunnel $(p=0.021)$.

Sensory deficits such as hypoesthesia in the plantar nerve dermatome were observed in $10(6.6 \%) \mathrm{FM}$ patient extremities and two (1.7\%) control subject extremities $(p=0.228)$; 10 (6.6\%) FM patient extremities and two $(1.7 \%)$ control subject extremities were positive for Tinel's sign $(p=0.228)$. There were no between-group differences in these variables. Additionally, there were no between-group differences in weakness of flexion of the toe or atrophy of the abductor hallucis muscle ( $p>0.05$ for all comparisons).

Tarsal tunnel syndrome was electrophysiologically diagnosed in 14 (9.2\%) FM patient extremities versus two (1.7\%) control subject extremities $(p=0.009)$. Deep peroneal neuropathy and peroneal neuropathy at the fibular head were electrophysiologically diagnosed in six (3.9\%) FM patient extremities versus one $(0.7 \%)$ control subject extremity and in four (2.6\%) FM patient extremities versus one $(0.7 \%)$ control subject extremities, respectively. No betweengroup differences were observed in these variables (Table 2).

\begin{tabular}{|c|c|c|c|}
\hline & Fibromyalgia patients $(n=76)$ & Healthy control subjects $(n=60)$ & \\
\hline & Mean \pm SD & Mean \pm SD & $p$ \\
\hline Age (year) & $39 . \pm 7.4$ & $38.6 \pm 8.2$ & 0.65 \\
\hline Height $(\mathrm{cm})$ & $159 \pm 4.4$ & $160 \pm 5.8$ & 0.74 \\
\hline Weight (kg) & $59.8 \pm 8.6$ & $60.7 \pm 9.8$ & 0.69 \\
\hline Body mass index $\left(\mathrm{kg} / \mathrm{m}^{2}\right)$ & $26.3 \pm 3.9$ & $27.1 \pm 4.9$ & 0.69 \\
\hline
\end{tabular}


Table 2. Clinical and electrophysiological characteristics of fibromyalgia patients and healthy control subjects

\begin{tabular}{|c|c|c|c|c|c|c|c|}
\hline & \multicolumn{3}{|c|}{$\begin{array}{l}\text { Fibromyalgia patient extremities } \\
\qquad(\mathrm{n}=152)\end{array}$} & \multicolumn{3}{|c|}{$\begin{array}{l}\text { Healthy control subject extremities } \\
\qquad(\mathrm{n}=120)\end{array}$} & \multirow[b]{2}{*}{$p$} \\
\hline & Sum & Right & Left & Sum & Right & Left & \\
\hline Paresthesia of the foot & 22 & 12 & 10 & 4 & 3 & 1 & $0.002 *$ \\
\hline Sensory deficits in the plantar nerve dermatome & 10 & 6 & 4 & 2 & 1 & 1 & 0.228 \\
\hline Local tenderness at the tarsal tunnel & 12 & 7 & 5 & 2 & 2 & 0 & $0.021^{*}$ \\
\hline Weakness of flexion of the toe & 3 & 2 & 1 & 1 & 1 & 0 & 0.438 \\
\hline Abductor hallucis muscle atrophy & 3 & 2 & 1 & 1 & 0 & 1 & 0.438 \\
\hline Positive Tinel's sign & 10 & 6 & 4 & 2 & 1 & 1 & 0.228 \\
\hline \multicolumn{8}{|l|}{ Electrophysiologically diagnosed } \\
\hline Deep peroneal neuropathy & 6 & 4 & 2 & 1 & 1 & 0 & 0.107 \\
\hline Peroneal neuropathy at the fibular head & 4 & 3 & 1 & 1 & 1 & 0 & 0.273 \\
\hline Tarsal tunnel syndrome & 14 & 8 & 6 & 2 & 1 & 1 & $0.009 *$ \\
\hline Total entrapment neuropathy & 24 & 15 & 9 & 4 & 3 & 1 & $0.001^{*}$ \\
\hline
\end{tabular}

Table 3. Nerve conduction studies of fibromyalgia patients and healthy control subjects

\begin{tabular}{|c|c|c|c|c|c|c|c|}
\hline & \multicolumn{2}{|c|}{$\begin{array}{l}\text { Fibromyalgia patient extremities } \\
\qquad(\mathrm{n}=152)\end{array}$} & \multicolumn{2}{|c|}{$\begin{array}{l}\text { Healthy control subject extremities } \\
\qquad(\mathrm{n}=120)\end{array}$} & \multirow{3}{*}{$\frac{\text { Right }}{p}$} & \multirow{3}{*}{$\begin{array}{c}\text { Left } \\
p\end{array}$} & \multirow{3}{*}{ Normal } \\
\hline & Right & Left & Right & Left & & & \\
\hline & Mean \pm SD & Mean \pm SD & Mean \pm SD & Mean \pm SD & & & \\
\hline \multicolumn{8}{|l|}{ Tibial (motor) } \\
\hline $\mathrm{TL}(\mathrm{m} / \mathrm{sec})$ & $4.9 \pm 0.6$ & $4.8 \pm 0.72$ & $4.7 \pm 0.6$ & $4.7 \pm 0.5$ & 0.54 & 0.68 & $<5.3$ \\
\hline $\mathrm{V}_{\mathrm{mot}}(\mathrm{m} / \mathrm{sec})$ & $45.7 \pm 4.5$ & $45.1 \pm 4.6$ & $46.5 \pm 4.3$ & $47.1 \pm 4.5$ & 0.48 & 0.13 & $>41.1$ \\
\hline CMAP (mV) & $10.9 \pm 4.2$ & $10.8 \pm 4.3$ & $10.5 \pm 3.9$ & $10.6 \pm 4.0$ & 0.52 & 0.60 & $>5$ \\
\hline \multicolumn{8}{|l|}{ Peroneal (motor) } \\
\hline TL (ms) & $4.6 \pm 0.7$ & $4.5 \pm 0.6$ & $4.5 \pm 0.7$ & $4.6 \pm 0.6$ & 0.67 & 0.68 & $<5.4$ \\
\hline $\mathrm{V}_{\mathrm{mot}}(\mathrm{m} / \mathrm{s})$ & $49.6 \pm 3.3$ & $49.2 \pm 3.2$ & $50.5 \pm 4.0$ & $50.7 \pm 3.9$ & 0.52 & 0.28 & $>43.5$ \\
\hline CMAP $(\mathrm{mV})$ & $4.9 \pm 0.6$ & $5.1 \pm 0.7$ & $5.2 \pm 0.6$ & $5.4 \pm 0.7$ & 0.52 & 0.53 & $>2$ \\
\hline \multicolumn{8}{|l|}{ Sural (sensory) } \\
\hline $\mathrm{V}_{\text {sens }}(\mathrm{m} / \mathrm{s})$ & $37.5 \pm 2.8$ & $37.4 \pm 2.7$ & $38.2 \pm 2.9$ & $38.4 \pm 3.0$ & 0.34 & 0.23 & $>32.1$ \\
\hline $\operatorname{SNAP}(\mu \mathrm{V})$ & $14.6 \pm 7.4$ & $14.9 \pm 7.3$ & $14.2 \pm 7.0$ & $15.2 \pm 7.4$ & 0.58 & 0.64 & $>5$ \\
\hline \multicolumn{8}{|c|}{ Medial plantar (motor) } \\
\hline TL (ms) & $4.2 \pm 0.6$ & $4.3 \pm 0.6$ & $4.0 \pm 0.6$ & $4.2 \pm 0.6$ & 0.51 & 0.63 & $\leq 5.4$ \\
\hline CMAP (mV) & $6.9 \pm 1.4$ & $6.8 \pm 1.4$ & $7.2 \pm 1.5$ & $7.1 \pm 1.3$ & 0.53 & 0.52 & $\geq 3.5$ \\
\hline \multicolumn{8}{|c|}{ Lateral plantar (motor) } \\
\hline TL (ms) & $4.8 \pm 0.7$ & $4.9 \pm 0.7$ & $4.7 \pm 0.6$ & $4.8 \pm 0.9$ & 0.67 & 0.68 & $\leq 6.3$ \\
\hline CMAP (mV) & $6.8 \pm 2.6$ & $6.8 \pm 2.0$ & $7.1 \pm 2.8$ & $7.0 \pm 3.2$ & 0.54 & 0.60 & $\geq 3.0$ \\
\hline \multicolumn{8}{|c|}{ Medial plantar (sensory) } \\
\hline$V_{\text {sens }}(\mathrm{m} / \mathrm{s})$ & $44.8 \pm 6.6$ & $43.4 \pm 7.2$ & $45.2 \pm 7.2$ & $44.8 \pm 8.1$ & 0.64 & 0.32 & $\leq 28.0$ \\
\hline $\operatorname{SNAP}(\mu \mathrm{V})$ & $3.4 \pm 1.9$ & $3.5 \pm 1.8$ & $3.5 \pm 2.1$ & $3.4 \pm 1.9$ & 0.67 & 0.65 & $\geq 2.0$ \\
\hline \multicolumn{8}{|c|}{ Lateral plantar (sensory) } \\
\hline $\mathrm{V}_{\text {sens }}(\mathrm{m} / \mathrm{s})$ & $31.2 \pm 4.6$ & $31.6 \pm 4.5$ & $32.7 \pm 4.5$ & $32.3 \pm 4.2$ & 0.24 & 0.37 & $\leq 22.9$ \\
\hline $\operatorname{SNAP}(\mu \mathrm{V})$ & $1.9 \pm 0.6$ & $2.0 \pm 0.6$ & $2.1 \pm 0.8$ & $2.2 \pm 0.7$ & 0.42 & 0.43 & $\geq 1.0$ \\
\hline
\end{tabular}

SD: Standard deviation; TL: Terminal motor latency; $V_{\text {mot: }}$ Motor conduction velocity; CMAP: Compound muscle action potential; $V_{\text {sens: }}$ Sensory conduction velocity; SNAP: Sensory nerve action potential; Student's t-test was used. 
Table 4. Shortest $\mathrm{F}$ wave latencies and $\mathrm{H}$-reflexes in fibromyalgia patients and healthy control subjects

\begin{tabular}{|c|c|c|c|c|c|c|c|}
\hline & \multicolumn{2}{|c|}{$\begin{array}{l}\text { Fibromyalgia patient } \\
\text { extremities }(\mathrm{n}=152)\end{array}$} & \multicolumn{2}{|c|}{$\begin{array}{l}\text { Healthy control subject } \\
\text { extremities }(n=120)\end{array}$} & \multirow[b]{2}{*}{ Right } & \multirow[b]{2}{*}{ Left } & \multirow[b]{2}{*}{ Normal } \\
\hline & Right & Left & Right & Left & & & \\
\hline & Mean \pm SD & Mean \pm SD & Mean \pm SD & Mean \pm SD & $p$ & $p$ & $p$ \\
\hline Peroneal $F_{\min }$ & $44.6 \pm 3.8$ & $45.2 \pm 4.2$ & $43.5 \pm 3.8$ & $44.8 \pm 5.2$ & 0.32 & 0.63 & $<52.3$ \\
\hline Tibial $F_{\min }$ & $45.2 \pm 4.8$ & $44.8 \pm 4.5$ & $44.2 \pm 4.0$ & $44.6 \pm 4.2$ & 0.33 & 0.88 & $<53.4$ \\
\hline Tibial H-reflex & $30.6 \pm 2.8$ & $31.2 \pm 4.2$ & $29.6 \pm 3.8$ & $29.8 \pm 3.6$ & 0.34 & 0.30 & $<31.8$ \\
\hline
\end{tabular}

The results of NCSs are shown in Table 3, and Table 4 presents minimum $\mathrm{F}$ wave latency $\left(F_{\min }\right)$ and H-reflex data; none of the data differed significantly between the two groups. When FM patient extremities were examined individually, the following abnormalities were identified: 14 yielded data suggestive of TTS (five had prolonged terminal motor latencies [TLs] and slow sensory nerve conduction velocities [NCVs] in the absence of sensory nerve action potentials [SNAPs] in the medial plantar nerves, four had slow sensory NCVs with a decrease in SNAPs in the lateral plantar nerves, three had slow sensory NCVs with a decrease in SNAPs in the medial plantar nerve, and two had a prolonged TL and slow sensory NCV with a decrease in SNAP in the lateral plantar nerve), six yielded data suggestive of deep peroneal neuropathy (four had prolonged TL and $F_{\min }$ values in the peroneal nerves, and two had prolonged TLs and decreased compound muscle action potential [CMAP] amplitudes in the peroneal nerves), four yielded data suggestive of peroneal nerve entrapment at the fibular head (decreased motor conduction velocity values, normal TLs and $F_{\min }$, but CMAP amplitudes at the lower limit of normal group values after stimulation of the peroneal nerve at the fibular head), and one yielded data suggestive of an S1 radiculopathy (prolonged tibial $\mathrm{F}$ wave latency, prolonged H-reflex, normal peroneal nerve conduction, normal peroneal $\mathrm{F}$ waves, and decreased sural SNAP amplitudes). No extremities exhibited multiple nerve involvement. There was no evidence of generalized polyneuropathy in any subject.

When control subject extremities were evaluated individually, the following abnormalities were identified: four had abnormal findings in terms of peroneal nerve conduction (prolonged TLs), one yielded data suggestive of lumbar radiculopathy (prolonged peroneal $\mathrm{F}$ wave latency, normal tibial, peroneal, and sural nerve conduction, normal tibial $\mathrm{F}$ wave latency, and normal H-reflex), and two yielded data suggestive of TTS (slow sensory NCV with no SNAP in the medial plantar nerve).

\section{DISCUSSION}

Our results showed that the frequency of paresthesia (reported as numbness and tingling sensations of the foot), local tenderness at the tarsal tunnel, and TTS were significantly higher in FM patients than those in control subjects. Sensory deficits such as hypoesthesia in the plantar nerve dermatome and a positive Tinel's sign tended to be more prevalent in FM patients than those in control subjects, but these differences were not statistically significant. Taking these findings into account, we suggest that there may be typical clinical features of TTS common in FM patients.

Tarsal tunnel syndrome of the foot is analogous to CTS of the wrist. Although some studies have reported high frequencies of CTS in FM patients, ${ }^{9-11}$ no previous report has addressed the frequency of TTS in FM patients. In our study, TTS was detected electrophysiologically in 14 (9.2\%) extremities of $79 \mathrm{FM}$ patients and two extremities of 60 control subjects. Compared to control subjects, FM patients had a higher frequency of TTS $(p<0.05)$. Moreover, deep peroneal neuropathy was detected electrophysiologically in six FM patient extremities and one control subject extremity. Peroneal neuropathy at the fibular head was detected electrophysiologically in four FM patient extremities and one control subject extremity. Overall, 24 (15.7\%) FM patient extremities yielded electrophysiological findings 
suggestive of focal entrapment neuropathy. Importantly, the frequency of focal entrapment neuropathy in FM patient extremities was significantly higher than that in control subject extremities. This finding may be related to weight gain, musculoskeletal pain, biomechanical factors secondary to hypermobility, and increased sensitization as a result of central sensitization. ${ }^{2,3}$

The etiologies of nerve entrapments are likely multifactorial. Simple nerve compression secondary to weight loss, prolonged periods of immobilization, incorrect positioning of body parts, and repetitive motion are likely to play a role in nerve entrapment. As the precise mechanism by which FM causes peripheral nerve entrapments is still not clear, we believe that prolonged periods of immobilization and incorrect positioning of body parts are some of the major reasons of nerve entrapment in FM. Due to generalized musculoskeletal pain, FM patients have significant limitations in functional mobility, namely difficulties in goal-directed movements. ${ }^{12,13}$ Pain observed in FM patients is considered to be due to nociceptive and neuropathic dysregulation of the central nervous system as well as psychological pathology. ${ }^{14}$ Thus, pain avoidance can lead to prolonged immobility in these patients. Fatigue and sleep disturbance are also characteristic symptoms of FM and influence mobility. ${ }^{1}$ These functional disabilities in FM patients could contribute to nerve entrapments. Taken together, we suggest that widespread musculoskeletal pain, fatigue, and sleep disturbances may lead to the immobilization of FM patients and subsequent vulnerability to entrapment neuropathy. However, the exact nature of the association between FM and entrapment neuropathies requires further investigation.

A better approach to differentiate paresthesia and other sensory complaints from compression neuropathies is needed in FM patients. ${ }^{15}$ Reported frequencies of paresthesia in FM patients $(>60 \%)$ are quite remarkable. ${ }^{16,17}$ In FM patients, increased subjective sensitivity in response to multisensory stimulation and reduced tolerance to non-nociceptive sensory stimulation may interfere with the interpretation of paresthetic symptoms. ${ }^{18}$ Additional electroneuromyography and NCSs may thus be helpful for distinguishing paresthesia and other sensory symptoms from entrapment neuropathies in FM patients. ${ }^{15}$
Our study had some limitations. First, we did not perform NCSs in upper extremities. Some outcomes may be biased due to lack of blinding. However, these outcomes were not formally assessed making it impossible for a blinded adjudication committee to eliminate the bias. Second, the patients recruited in this study were diagnosed with FM according to the ACR 1990 criteria. Previous diagnostic criteria were adopted in our study to differentiate between the objective clinical findings in FM patients and control subjects without FM, but further studies are needed based on the latest diagnostic criteria. Furthermore, our study may have selection bias; due to strict exclusion criteria, we may have failed to include patients with severe polyneuropathy and concomitant TTS. In the future, detailed NCSs on both the upper and lower extremities of a large study population may yield more information on the role of PNS in FM patients. However, to the best of our knowledge, this is the first study to compare the clinical features of TTS between FM patients and control subjects with paresthesia and sensory deficits observed during physical examination.

In conclusion, our major findings were that the prevalence of paresthesia of the foot and local tenderness at the tarsal tunnel were significantly higher in FM patients than that in healthy control subjects. Furthermore, TTS was statistically more frequent in FM patients than that in control subjects. Finally, we did not find any evidence of generalized abnormality of the PNS playing a role in the pathogenesis of FM. As the presence of TTS may be masked by the symptoms of FM, individuals with both paresthesia of the foot and FM should be carefully examined for the accompanying TTS so that appropriate and effective therapeutic interventions can be applied. In addition, electrodiagnostic tests may be helpful for the detection of undiagnosed TTS.

\section{Declaration of conflicting interests}

The authors declared no conflicts of interest with respect to the authorship and/or publication of this article.

\section{Funding}

The authors received no financial support for the research and/or authorship of this article. 


\section{REFERENCES}

1. Wolfe F, Smythe HA, Yunus MB, Bennett RM, Bombardier C, Goldenberg DL, et al. The American College of Rheumatology 1990 Criteria for the Classification of Fibromyalgia. Report of the Multicenter Criteria Committee. Arthritis Rheum 1990;33:160-72.

2. Shookster L, Falke GI, Ducic I, Maloney CT Jr, Dellon AL. Fibromyalgia and Tinel's sign in the foot. $\mathrm{J}$ Am Podiatr Med Assoc 2004;94:400-3.

3. Sluka KA, Clauw DJ. Neurobiology of fibromyalgia and chronic widespread pain. Neuroscience 2016;338:114-29.

4. Cagnie B, Coppieters I, Denecker S, Six J, Danneels L, Meeus M. Central sensitization in fibromyalgia? A systematic review on structural and functional brain MRI. Semin Arthritis Rheum 2014;44:68-75.

5. Nacir B, Genc H, Duyur Cakit B, Karagoz A, Erdem HR. Evaluation of upper extremity nerve conduction velocities and the relationship between fibromyalgia and carpal tunnel syndrome. Arch Med Res 2012;43:369-74.

6. Patel AT, Gaines K, Malamut R, Park TA, Toro DR, Holland N; American Association of Neuromuscular and Electrodiagnostic Medicine. Usefulness of electrodiagnostic techniques in the evaluation of suspected tarsal tunnel syndrome: an evidence-based review. Muscle Nerve 2005;32:236-40.

7. Oh SJ, Sarala PK, Kuba T, Elmore RS. Tarsal tunnel syndrome: Electrophysiological study. Ann Neurol 1979;5:327-30.

8. Jo YS, Na SJ. Tarsal tunnel syndrome in patients with fibromyalgia. J Neurol Sci 2017;381:469.

9. Cimmino MA, Parisi M, Moggiana G, Accardo S. The association between fibromyalgia and carpal tunnel syndrome in the general population. Ann Rheum Dis 1996;55:780.
10. Sarmer S, Yavuzer G, Küçükdeveci A, Ergin S. Prevalence of carpal tunnel syndrome in patients with fibromyalgia. Rheumatol Int 2002;22:68-70.

11. Perez-Ruiz F, Calabozo M, Alonso-Ruiz A, Herrero A, Ruiz-Lucea E, Otermin I. High prevalence of undetected carpal tunnel syndrome in patients with fibromyalgia syndrome. J Rheumatol 1995;22:5014.

12. Jones KD, Sherman CA, Mist SD, Carson JW, Bennett RM, Li F. A randomized controlled trial of 8-form Tai chi improves symptoms and functional mobility in fibromyalgia patients. Clin Rheumatol 2012;31:1205-14.

13. Björnsdóttir SV, Jónsson SH, Valdimarsdóttir UA. Functional limitations and physical symptoms of individuals with chronic pain. Scand J Rheumatol 2013;42:59-70.

14. Nielsen LA, Henriksson KG. Pathophysiological mechanisms in chronic musculoskeletal pain (fibromyalgia): the role of central and peripheral sensitization and pain disinhibition. Best Pract Res Clin Rheumatol 2007;21:465-80.

15. Borg-Stein J. Management of peripheral pain generators in fibromyalgia. Rheum Dis Clin North Am 2002;28:305-17.

16. Simms RW, Goldenberg DL. Symptoms mimicking neurologic disorders in fibromyalgia syndrome. J Rheumatol 1988;15:1271-3.

17. Balbaloglu O, Tanik N, Alpayci M, Ak H, Karaahmet E, Inan LE. Paresthesia frequency in fibromyalgia and its effects on personality traits. Int $J$ Rheum Dis 2018;21:1343-9.

18. López-Solà M, Pujol J, Wager TD, Garcia-Fontanals A, Blanco-Hinojo L, Garcia-Blanco S, et al. Altered functional magnetic resonance imaging responses to nonpainful sensory stimulation in fibromyalgia patients. Arthritis Rheumatol 2014;66:3200-9. 